\title{
A SUPERNOVA REMNANT IN THE DWARF HO IX
}

\author{
ULRICH HOPP \\ Universitätssternwarte München, D 81679 München, FRG \\ H.U. ZIMMERMANN \\ MPI für Extraterrestrische Physik, D 85740 Garching, FRG \\ MANFRED STICKEL \\ MPI für Astronomie, D 61117 Heidelberg, FRG \\ AND \\ CHRISTIAN HENKEL \\ MPI für Radioastronomie, D 53121 Bonn, FRG
}

The dwarf irregular galaxy Ho IX (DDO 66) is a satellite of the giant spiral M81. Triggered by an interaction with M81 (Hopp \& Schulte-Ladbeck, 1987 AA 187, 5), Ho IX may have recently undergone a burst of star formation. On very deep Calar Alto $3.5 \mathrm{~m}$ telescope $\mathrm{R}$ images, we detected a ring-like nebula in the northeastern part of Ho IX, which is situated near to the giant CO molecular cloud recently discussed by Henkel et al. (1993 AA $273, \mathrm{L15})$. The structure of the nebula resembles supernova remnants like CTB 80. No HI-features can be detected at its location, especially no hole. It shows a blue central point source $(\mathrm{R}=21.7)$. A Lucy deconvolution of the 0.9 " seeing images indicates further faint point sources, perhaps a small stellar cluster. An optical long slit spectrum of the nebula shows emission lines with ratios as in old supernova remnants while it excludes normal HII regions, planetary nebula, or the LMC X-1 type nebula. The position of the central object coincides with a bright and variable ROSAT point source which was already detected by the Einstein spacecraft. The X-ray spectrum is rather steep and shows strong intrinsic absorption. The nature of the X-ray source and its possible connection to the optical nebula is not yet understood. Miller (1995, ApJ 446, L75) who independetly found the nebula interpretated his spectra as a supershell surrounding an $\mathrm{OB}$ association. Our optical nebula line ratios severely deviates from Miller's. Our ratios as the X-ray spectrum point more to a SNR at the M81 distance, most probably belonging to Ho IX. 